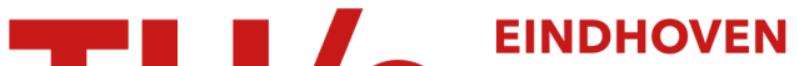 \\ UNIVERSITY OF \\ TECHNOLOGY
}

\section{Superfast fronts of impact ionization in initially unbiased layered semiconductor structures}

\section{Citation for published version (APA):}

Rodin, P., Ebert, U., Hundsdorfer, W., \& Grekhov, I. V. (2002). Superfast fronts of impact ionization in initially unbiased layered semiconductor structures. Journal of Applied Physics, 92(4), 1971-1980.

https://doi.org/10.1063/1.1494113

DOI:

10.1063/1.1494113

Document status and date:

Published: 01/01/2002

\section{Document Version:}

Publisher's PDF, also known as Version of Record (includes final page, issue and volume numbers)

\section{Please check the document version of this publication:}

- A submitted manuscript is the version of the article upon submission and before peer-review. There can be important differences between the submitted version and the official published version of record. People interested in the research are advised to contact the author for the final version of the publication, or visit the $\mathrm{DOI}$ to the publisher's website.

- The final author version and the galley proof are versions of the publication after peer review.

- The final published version features the final layout of the paper including the volume, issue and page numbers.

Link to publication

\section{General rights}

Copyright and moral rights for the publications made accessible in the public portal are retained by the authors and/or other copyright owners and it is a condition of accessing publications that users recognise and abide by the legal requirements associated with these rights.

- Users may download and print one copy of any publication from the public portal for the purpose of private study or research.

- You may not further distribute the material or use it for any profit-making activity or commercial gain

- You may freely distribute the URL identifying the publication in the public portal.

If the publication is distributed under the terms of Article 25fa of the Dutch Copyright Act, indicated by the "Taverne" license above, please follow below link for the End User Agreement:

www.tue.nl/taverne

Take down policy

If you believe that this document breaches copyright please contact us at:

openaccess@tue.nl

providing details and we will investigate your claim. 


\title{
Superfast fronts of impact ionization in initially unbiased layered semiconductor structures
}

\author{
P. Rodin ${ }^{\text {a) }}$ \\ Centrum voor Wiskunde en Informatica, Postbus 94079, 1090 GB Amsterdam, The Netherlands \\ and Institute for Theoretical Physics, TU Berlin, Hardenbergstrasse 36, 10623 Berlin, Germany \\ U. Ebert and W. Hundsdorfer \\ Centrum voor Wiskunde en Informatica, Postbus 94079, 1090 GB Amsterdam, The Netherlands \\ I. V. Grekhov \\ Ioffe Physicotechnical Institute, Politechnicheskaya 26, 194021, St. Petersburg, Russia
}

(Received 22 February 2002; accepted for publication 23 May 2002)

\begin{abstract}
A mode of impact ionization breakdown of a $p-n$ junction is suggested: We demonstrate that when a sufficiently sharp voltage ramp is applied in reverse direction to an initially unbiased equilibrium $p^{+}-n-n^{+}$structure, after some delay the system will reach a high conductivity state via the propagation of a superfast impact ionization front. The front travels towards the anode with a velocity $v_{f}$ several times larger than the saturated drift velocity of electrons $v_{s}$ leaving a dense electron-hole plasma behind. The excitation of the superfast front corresponds to the transition from the common avalanche breakdown of a semiconductor structure to a collective mode of streamer-like breakdown. We propose that similar fronts can be excited not in layered structures but in plain bulk samples without $p-n$ junctions. Our numerical simulations apply to a $\mathrm{Si}$ structure with typical thickness of $W \sim 100 \mu \mathrm{m}$ switched in series with a load $R \sim 100 \Omega$, with a voltage ramp of $A>10^{12} \mathrm{~V} / \mathrm{s}$ applied to the whole system. Our simulations show that first there is a delay of about $1 \mathrm{~ns}$ during which the voltage reaches a value of several kilovolts. Then, as the front is triggered, the voltage abruptly breaks down to several hundreds of volts within $\sim 100 \mathrm{ps}$. This provides a voltage ramp of up to $\sim 2 \times 10^{13} \mathrm{~V} / \mathrm{s}$ hence up to 10 times sharper than the externally applied ramp. We unravel the source of initial carriers which trigger the front, explain the origin of the time delay in triggering the front, and we identify the mechanism of front propagation. (c) 2002 American Institute of Physics. [DOI: 10.1063/1.1494113]
\end{abstract}

\section{INTRODUCTION}

Traveling front patterns are common nonlinear phenomena in various spatially extended systems far from equilibrium. ${ }^{1-3}$ In gases and solids exposed to a strong external electrical field, a conducting region might penetrate an essentially nonconducting region in the form of a field driven impact ionization front. The front is characterized by a space charge that screens the electric field from the highly ionized plasma region. Depending on the initial degree of ionization, on the polarity of the applied voltage, and on the importance of nonlocal ionization reactions, the ionization process takes place inside or in the vicinity of the screening front. ${ }^{4-17}$ The structure of the impact ionization front transverse to the direction of front propagation depends on the experimental setup, e.g., on the form of the electrodes and other outer boundaries, the voltage, etc.. In long narrow glass tubes filled with gas, planar ionization fronts have been observed, ${ }^{6}$ whereas in many other discharges in gases or solids the ionization front propagates at the tip of a long and narrow highly conducting channel, the so-called streamer, ${ }^{4-16}$ and the propagation process is essentially three-dimensional. Ionization fronts of various shapes-plane fronts, finger-shaped

a) On leave from Ioffe Physicotechnical Institute, Politechnicheskaya 26, 194021, St. Petersburg, Russia; electronic mail: rodin@physik.tu-berlin-de streamers, concentric fronts in samples with Corbino geometry, etc- - have been observed in semiconductors, including bulk semiconductors, ${ }^{7}$ semiconductor films of different contact geometries, ${ }^{3}$ and layered structures of semiconductor devices. ${ }^{18-20}$

In this article we deal with superfast impact ionization fronts in layered semiconductor structures that propagate many times faster than the saturated drift velocity of free carriers $v_{s}$ and create a dense electrically screened electronhole plasma. The superfast front velocity indicates that the front propagation represents a collective phenomenon that makes this mode of impact ionization breakdown important from a fundamental point of view, and also provides the basis of numerous applications. This spectacular wavelike mode of the impact ionization breakdown of layered $p^{+}-n-n^{+}$structures was discovered several decades ago. ${ }^{21,22}$ It underlies operation of well-known trapped plasma avalanche triggered transit diodes ${ }^{22}$ (TRAPATT) used as microwave generators. Later it was found that a similar wavelike breakdown can be achieved in much larger structures with kilovolt $p-n$ junctions and large $\left(\sim 10^{-2} \mathrm{~cm}^{2}\right)$ cross sections. ${ }^{23,24}$ For such structures, often coined as silicon avalanche sharpening diodes, the output power per pulse is by 4 orders of magnitude higher than for TRAPATT diodes $\left(10^{7}\right.$ versus $10^{3} \mathrm{~W}$ ), and the main applications are in pulse power 
electronics. ${ }^{23-28}$ A TRAPATT diode is embedded in a microwave cavity and operated in a regime of periodic oscillations where the impact ionization fronts almost immediately follow each other, so that the nonequilibrium carriers from the previous front passage play an essential role in the excitation of the next front. In contrast, for power kilovolt structures the time interval between two subsequent front passages is large compared to the system relaxation time and each excitation of a front represents an independent event. ${ }^{29-34}$ For the solitary front passage, the wavelike mode of impact ionization breakdown takes the simplest and hence the most fundamental form. Here we shall focus entirely on this solitaryfront-passage regime.

Superfast impact ionization fronts have been found experimentally in $p^{+}-n-n^{+}$structures with kilovolt $p-n$ junctions that initially are subject to a reverse bias $V_{0}$ which is closely below the voltage of stationary avalanche breakdown $U_{a} \cdot{ }^{24-28}$ If the applied reverse voltage is further increased (for example, $V(t)=V_{0}+A t$ ) with a sufficiently high rate $A$, the impact ionization starts near the $p^{+}$layer, where the electrical field takes its maximal value, and develops into an impact ionization front that travels towards the $n^{+}$layer with a velocity $v_{f}>v_{s}$. It leaves a dense electronhole plasma behind with an electron concentration $N$ much higher than the doping level in the $n$ base $N_{d}$.

The main purpose of this article is to demonstrate that the initial bias $V_{0}$ is in fact not needed, and switching due to the propagation of a superfast front can be successfully achieved in initially unbiased structures for $V_{0}=0$. We present numerical simulations of this mode of impact ionization breakdown. We unravel the source of mobile carriers that initialize the impact ionization and that provide the background ionization needed for propagation with velocity $v_{f}>v_{s}$. We argue that the excitation of the superfast front generally corresponds to the transition from the common avalanche breakdown of a semiconductor structure to a collective mode of streamer-like breakdown and relate the impact ionization fronts in layered semiconductor structures to theoretical studies of streamer propagation in gases and solids. ${ }^{10-16}$ Based on our results, we propose that superfast fronts can be excited not only in layered structures but also in bulk semiconductors.

The paper is organized as follows. After introducing the semiconductor structure and the physical model in Sec. II, in Sec. III we present results of numerical simulations for one typical set of parameters and describe the main stages of the superfast switching process. In Sec. IV we discuss problems of an unreflected use of the continuum approximation for the carrier densities and our strategy to avoid them. Section V deals with the question of how the switching process depends on the structure length and the voltage ramp applied to the device. In Sec. VI, we survey alternative sources of initial carriers that can trigger the front and discuss our results.

\section{THE MODEL}

The excitation of impact ionization fronts has been observed in experiments in the large family of $\mathrm{Si}^{23,25,35,36}$ or $\mathrm{GaAs}^{37-39}$ layered semiconductor structures. Here we shall

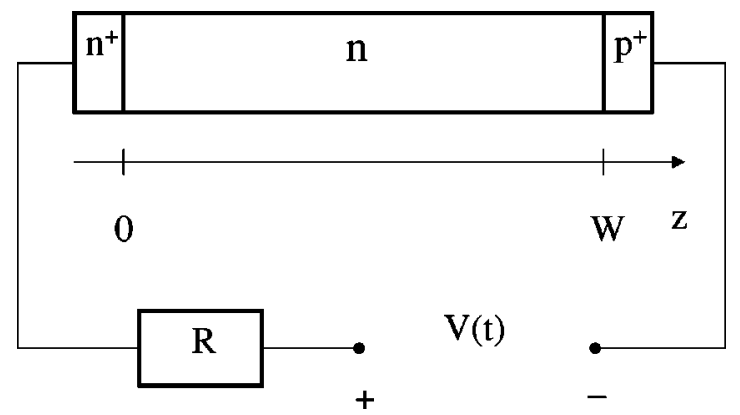

FIG. 1. Sketch of the $p^{+}-n-n^{+}$structure operated in an external circuit with load resistance $R$

concentrate on the basic case of a Si diode $p^{+}-n-n^{+}$structure with sharp $p^{+}-n$ and $n-n^{+}$transitions with the following typical parameters: the width of the $n$-base $W$ is 100 to $300 \mu \mathrm{m}$, the area $S$ of the cross section transverse to the direction of current is approximately 0.005 to $0.05 \mathrm{~cm}^{2}$, the doping levels are $N_{d} \approx 10^{14} \mathrm{~cm}^{-3}$ in the $n$-base, and $N_{a, d}^{+}$ $\approx 10^{19}-10^{20} \mathrm{~cm}^{-3}$ in the contact $p^{+}$and $n^{+}$layers, respectively. The device is connected to the voltage source $V(t)$ in series with the load resistance $R \sim 50-100 \Omega$ as sketched in Fig. 1, and is operated at room temperature. Note that we have chosen to put the $n^{+}$contact on the left hand side at $z=0$ in all figures and equations throughout the paper. This choice allows us to work with positive electrical fields for reverse bias.

The dynamics of charge carriers is described in the drift-diffusion approximation by the following standard set of continuity and transport equations:

$$
\begin{aligned}
& \frac{\partial n}{\partial t}-\nabla \cdot \frac{\mathbf{J}_{n}}{q}=G(n, p,|E|), \\
& \frac{\mathbf{J}_{n}}{q}=v_{n}(|E|) \hat{\mathbf{e}} n+D_{n} \boldsymbol{\nabla} n, \\
& \frac{\partial p}{\partial t}+\nabla \cdot \frac{\mathbf{J}_{p}}{q}=G(n, p,|E|), \\
& \frac{\mathbf{J}_{p}}{q}=v_{p}(|E|) \hat{\mathbf{e}} p-D_{p} \nabla p,
\end{aligned}
$$

together with the Poisson equation for the electric field

$$
\begin{aligned}
& \boldsymbol{\nabla} \cdot \mathbf{E}=\frac{q}{\epsilon \epsilon_{0}}\left[p-n+N_{d}(z)-N_{a}(z)\right], \\
& \mathbf{E}=-\boldsymbol{\nabla} \Phi, \quad \hat{\mathbf{e}}=\frac{\mathbf{E}}{|\mathbf{E}|} .
\end{aligned}
$$

Here $n$ and $p$ are concentrations of electrons and holes, respectively. $\Phi$ and $\mathbf{E}$ are electrical potential and field, $q>0$ is the elementary charge, and $\mathbf{J}_{n, p}$ are the current densities carried by electrons and holes, respectively. $\epsilon$ and $\epsilon_{0}$ are the permittivity of the material and the absolute permittivity, respectively. $D_{n, p}$ are the diffusion coefficients of electrons and holes. $N_{d}(z)$ and $N_{a}(z)$ are the concentration of donors and acceptors, respectively, which form the doping profile of the $p^{+}-n-n^{+}$structure along the $z$-direction. 
We here assume that all processes are uniform in the directions orthogonal to the cathode-anode direction $z$. We hence neglect transverse instabilities of a planar front ${ }^{30-34}$ and consider the problem in a one-dimensional approximation. Next, given the fact that physical processes relevant for the propagation of the ionization front develop in the bulk of the $n$ base, we restrict our consideration to the $n$-base area $0<z<W$. We impose mixed boundary conditions for concentrations $\partial_{z} n=0, p=0$ at $z=0$ and $\partial_{z} p=0, n=0$ at $z$ $=W$, that reflect the different effects of the contact $p^{+}$and $n^{+}$layers on electron and hole concentrations in the $n$ base. ${ }^{40}$ Since $N_{a, d}^{+} \gg N_{d}$ the voltage drop at the contact layers is also negligible.

We set the potential $\Phi(W, t) \equiv 0$ for all times $t$ and denote the voltage applied to the device with $U(t) \equiv \Phi(0, t)$ $>0$. This is related to the voltage $V(t)>0$ of the power source by Kirchhoff's equation

$$
\begin{aligned}
& V(t)=U(t)+R S J(t), \\
& J(t) \equiv J_{n}(z, t)+J_{p}(z, t)+\epsilon \epsilon_{0} \frac{\partial E(z, t)}{\partial t},
\end{aligned}
$$

where $J(t)$ is the total (conductive and displacement) current density in the system that is conserved in space $\left(\partial_{z} J=0\right)$ in one-dimensional systems, $S$ is the area of the cross section, and $E \equiv E_{z}$. Averaging Eq. (5) over the system length, we represent Eq. (4) as ${ }^{3,41}$

$$
\begin{aligned}
& R C \frac{d U}{d t}=V(t)-U-\frac{R S}{W} \int_{0}^{W}\left[J_{n}(z, t)+J_{p}(z, t)\right] d z, \\
& C=\frac{\epsilon \epsilon_{0} S}{W},
\end{aligned}
$$

where $C$ is the intrinsic capacitance of the semiconductor structure.

The applied voltage $V(t)$ typically represents a sinusoidal pulse $V(t)=V_{0}+V_{1} \sin (2 \pi t / T)$, or a combination of such a pulse for $t<T / 4$ with a plateau $V=V_{0}+V_{1}$ for $t$ $>T / 4$. Since the duration of the fast impact ionization breakdown is usually smaller than $T / 4$, in most cases the applied voltage can be approximated by the linear function

$$
V(t)=V_{0}+A t, \quad A \equiv 2 \pi V_{1} / T,
$$

where $A$ is the voltage ramp.

For the dependencies of electron and hole velocities $v_{s}$ and $v_{p}$ on the electrical field $E$ in $\mathrm{Si}$, we use the simplified version of the approximation suggested in Ref. 42:

$$
v_{n}(|E|)=v_{s} \frac{|E|}{E_{s n}+|E|}, \quad v_{p}(|E|)=v_{s} \frac{|E|}{E_{s p}+|E|},
$$

where

$$
\begin{aligned}
& v_{s}=10^{7} \mathrm{~cm} / \mathrm{s}, \\
& E_{s n}=8.0 \times 10^{3} \mathrm{~V} / \mathrm{cm}, \quad E_{s p}=2.32 \times 10^{4} \mathrm{~V} / \mathrm{cm} .
\end{aligned}
$$

These approximations describe the monotonic transition from the low-field ohmic regime $v_{n, p}(E)=\mu_{n, p} E$ (low-field mobilities are given by $\left.\mu_{n, p}=v_{s} / E_{s n, s p}\right)$ to the high-field transport with saturated drift velocity $v_{s}$ due to the scattering of carriers on optical phonons. Diffusion coefficients are determined by the Einstein relation $D_{n, p} / \mu_{n, p}=k T / q$, where $T$ is the temperature and $k$ is Boltzmann's constant. ${ }^{43}$

The term $G(n, p,|E|)$ describes the generation of charge carriers due to impact ionization both by electrons and holes. It is chosen as

$$
\begin{gathered}
G(n, p,|E|)=\alpha_{n}(|E|) v_{n}(|E|) n \Theta\left(n-n_{\text {cut }}\right) \\
+\alpha_{p}(|E|) v_{p}(|E|) p \Theta\left(p-p_{\text {cut }}\right), \\
\alpha_{n}(|E|) \equiv \alpha_{n s} e^{-b_{n} /|E|}, \quad \alpha_{n}(|E|) \equiv \alpha_{p s} e^{-b_{p} /|E|},
\end{gathered}
$$

where $\Theta(x)$ is the step function, and the impact ionization coefficients and the characteristic fields are given by ${ }^{44}$

$$
\begin{aligned}
& \alpha_{n s}=7.4 \times 10^{5} \mathrm{~cm}^{-1}, \quad \alpha_{p s}=7.25 \times 10^{5} \mathrm{~cm}^{-1}, \\
& b_{n}=1.1 \times 10^{6} \mathrm{~V} / \mathrm{cm}, \quad b_{p}=2.2 \times 10^{6} \mathrm{~V} / \mathrm{cm} .
\end{aligned}
$$

The cutoffs $n_{\text {cut }}$ and $p_{\text {cut }}$ have been introduced in Eq. (10) to exclude unphysical effects that otherwise appear for concentrations below the limits of the continuum approximation. The necessity and validity of these cutoffs will be discussed in Sec. IV.

The electrical fields relevant to the propagation of impact ionization fronts correspond to the interval $2 \times 10^{5}-4$ $\times 10^{5} \mathrm{~V} / \mathrm{cm}$. In this interval the impact ionization coefficients [Eq. (11)] rapidly increase with electrical field. The field $E_{a} \approx 2 \times 10^{5} \mathrm{~V} / \mathrm{cm}$ can be considered as an effective threshold of the impact ionization. Since $E_{s} \ll E_{a}$, the drift velocities are saturated in the impact ionization region.

On a nanosecond time scale recombination can be neglected. Although the diffusion term is taken into account in the transport equations (1) and (2), the diffusion turns out to be negligible compared to the drift and has no impact on our results.

For the numerical simulation a uniform space-time grid has been selected. The spatial discretization is based on a conservative formulation, in terms of fluxes describing the inflow and outflow over cells $[x-\Delta x / 2, x+\Delta x / 2]$, where $\Delta x$ is the grid width in space. The number of grid points used in these simulations was of the order of several thousands both in time and space, to obtain sufficiently accurate numerical results. The diffusive fluxes have been approximated in a standard fashion ${ }^{45}$ with second order accuracy. For the convective fluxes a third order upwind biased formula has been chosen in order to reduce the numerical oscillations that are common with second order central fluxes. Time discretization is based on a second order backward differentiation formula. The temporal backward differentiation formula gives an implicit system that is solved at each time step. For reasons of accuracy the time step $\Delta t$ is chosen small compared to $\Delta x / v_{s}$, where $v_{s}$ is the upper bound of the drift (convective) velocity, and with such small step size the implicit system can be solved by a straightforward functional iteration. Details on these spatial and temporal discretizations can be found in Ref. 46. 

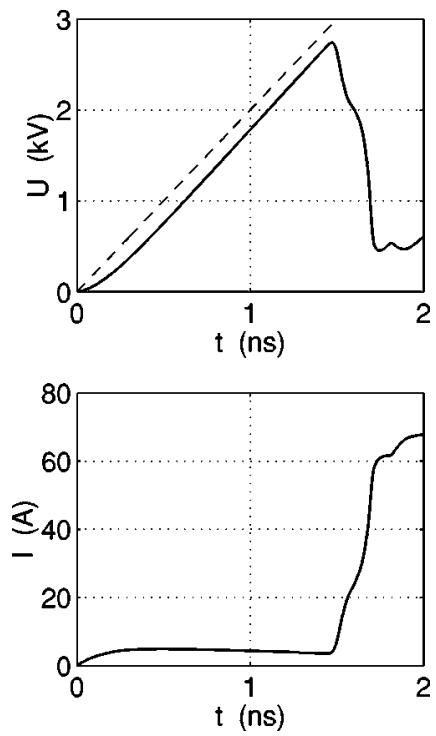

FIG. 2. Voltage at the structure $U(t)$ (solid line in the upper panel) and current $I(t)=S J(t)$ (in the lower panel) during the switching process. The dashed line in the upper panel denotes the externally applied voltage $V(t)$. The shown quantities are related through Ohm's law $V=U+R I$. Parameters: $W=150 \mu \mathrm{m}, \quad S=0.02 \mathrm{~cm}^{2}, \quad N_{d}=10^{14} \mathrm{~cm}^{-3}, \quad N_{a}=0, \quad V_{0}=0, A$ $=2.0 \mathrm{kV} / \mathrm{ns}, R=50 \Omega$, and $n_{\text {cut }}=p_{\text {cut }}=10^{9} \mathrm{~cm}^{-3}$.

\section{TRIGGERING AND PROPAGTION OF THE IMPACT IONIZATION FRONT}

In this section we present numerical solutions of our model for a typical set of parameters: the width of the $n$ base is taken to be $W=150 \mu \mathrm{m}$, its doping levels are $N_{d}$ $=10^{14} \mathrm{~cm}^{-3}$ and $N_{a}=0$, and its transverse area is $S$ $=0.02 \mathrm{~cm}^{2}$. The resistance of the load is $R=50 \Omega$. The voltage ramp is $A=2 \mathrm{kV} / \mathrm{ns}$, the initial voltage $V_{0}=0$. The cutoff at low concentrations is taken as $n_{\text {cut }}=p_{\text {cut }}=10^{9} \mathrm{~cm}^{-3}$.

The basic features of the numerical solution for these parameters are summarized in Fig. 2, which shows the voltage at the device $U(t)$ and the total current through the device $I(t)=S J(t)$. Up to the time $t \approx 1.5 \mathrm{~ns}$, the voltage $U(t)$ follows the increase of the applied voltage $V(t)$ and reaches the value $U \approx 3 \mathrm{kV}$, which exceeds the voltage of stationary breakdown ${ }^{18} U_{a} \approx 1.7 \mathrm{kV}$ by a factor of nearly 2 . Within the next approximately $230 \mathrm{ps}$, it drops to the residual voltage $U_{a} \approx 450 \mathrm{~V}$, so that suddenly almost the complete $V(t)$ is applied to the external load $R$. The abrupt decay of $U(t)$ after $t \approx 1.5 \mathrm{~ns}$ shows a "fine structure" at $t \approx 1.6 \mathrm{~ns}$, reflecting a change in the internal breakdown dynamics. The average voltage drop $|d U / d t| \approx 10 \mathrm{kV} / \mathrm{ns}$ during the switching indicates a substantial sharpening of the externally applied pulse $V(t)$.

In the following subsections we discuss the different stages of the internal dynamics that underlie this switching process. The corresponding spatial profiles at different time steps are shown in Fig. 3.

\section{A. The latent stage [Fig. 3(a), $0<t<1.47 \mathrm{~ns}$ ]}

Initially the $n$ base is neutral since the major carriers (electrons) compensate the space charge of the donors: $n$ $\approx N_{d}=10^{14} \mathrm{~cm}^{-3}$. At room temperature, the concentration $p \approx N_{T} \approx 10^{5 \ldots 6} \mathrm{~cm}^{-3}$ of the minor carriers (holes) is much (a)
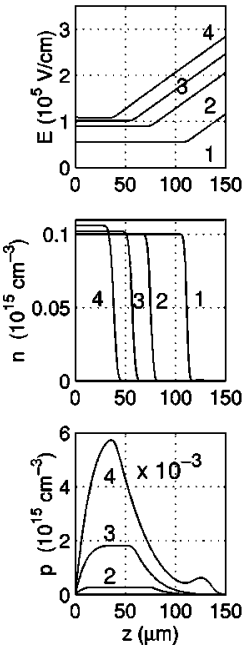

(b)
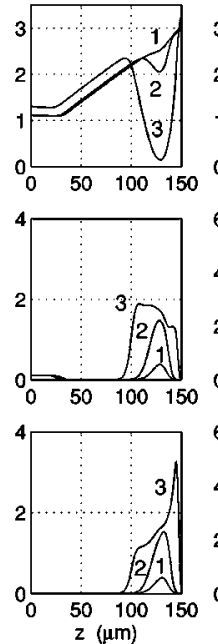

(c)
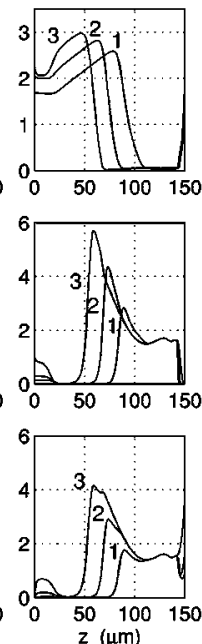

(d)

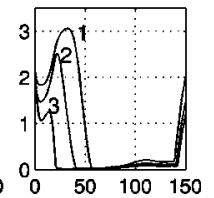

FIG. 3. The internal dynamics leading to the external characteristics of Fig. 2. Shown are the spatial profiles of the electrical field $E(x, t)$ and electron and hole concentrations $n(x, t), p(x, t)$ in the $n$ base $(0 \leqslant z \leqslant W=150 \mu \mathrm{m})$ at different times: (a) propagation of the extraction front and impact ionization in the neutral layer at times $t=0.6,1.0,1.2$, and $1.4 \mathrm{~ns}$ (curves 1, 2, 3, and 4, respectively); (b) nucleation of electron-hole plasma and triggering of the impact ionization front at times $t=1.47,1.49$, and 1.54 ns (curves 1 , 2 , and 3 ); (c) propagation of the impact ionization front at $t=1.59,1.63$, and $1.66 \mathrm{~ns}$ (curves 1, 2, and 3); (d) final stage of the switching: collision of impact ionization and extraction fronts, curves 1,2 , and 3 correspond to $t$ $=1.68,1.70$, and $1.73 \mathrm{~ns}$, respectively. All parameters as in Fig. 2. We note that the scale of the hole density $p$ is a factor of $10^{-3}$ smaller (i.e, $10^{12} \mathrm{~cm}^{-3}$ ) in column a and that we nevertheless skip curve 1 in this panel, since the the hole density at $t=0.6 \mathrm{~ns}$ vanishes even on this enlarged scale.

lower than $N_{d}$ and can be neglected. As the voltage applied to the device increases, the homogeneously distributed electrons drift towards the $n^{+}$contact where they are extracted from the $n$ base. The moving electron package leaves naked donors behind. Consequently, the device splits into a $d e-$ pleted region, which is free of major carriers, and a neutral region, where the major carriers are still present. The slope of the electrical field in the depleted region is determined by the space charge of naked donors: $d E / d z=q N_{d} / \epsilon \epsilon_{0}$. The electric field $E_{0}(t)$ in the neutral region is constant in space: $d E_{0} / d z=0$. In the following, we will refer to the boundary between these two regions as to the extraction front. The velocity of the extraction front is the electron drift velocity $v_{n}\left(E_{0}\right)$ corresponding to the electrical field $E_{0}$ in the neutral layer. It is bounded from above by the saturated drift velocity $v_{s}$.

Although the maximum value of the electrical field that is reached at the right boundary, overcomes the effective threshold $E_{a} \approx 2 \times 10^{5} \mathrm{~V} / \mathrm{cm}$ of impact ionization is Si for $t$ $>1 \mathrm{~ns}$, the ionization in the depleted region does not develop due to the absence of initial carriers. Rather, with an increase of the applied voltage a moderate impact ionization by electrons starts in the neutral region; despite the relatively low electrical field $E_{0}$, the ionization reaction develops due to the high electron concentration $n \approx N_{d}$. Although the total amount of generated free carriers is much smaller than $N_{d}$, a substantial concentration of holes $p \sim 10^{12} \mathrm{~cm}^{-3}$, much bigger than the thermal ionization $N_{T}$, is generated in the neu- 
tral region. The holes move to the right and eventually penetrate the region of high electric field near the $p^{+}$contact.

\section{B. Triggering of the impact ionization front [Fig. 3(b), $1.47 \mathrm{~ns}<t<1.60 \mathrm{~ns}]$}

As soon as drifting holes reach high electrical fields in the depleted region, the impact ionization by holes becomes efficient [curve 4 on Fig. 3(a) shows the beginning of the avalanche multiplication in the depleted region]. The generated electrons and holes in the high field region then multiply further and eventually form the nucleus of an electron-hole plasma capable of screening the electrical field [cf. curve 2 in Fig. 3(b)]. Since the concentration of holes that initialize the impact ionization decreases towards the right, the multiplication process does not start exactly at the right boundary of the $n$ base where the electric field is maximal. But within approximately $50 \mathrm{ps}$, the generated electron-hole plasma completely screens the electric field inside the nucleus. The charge generation by impact ionization continues on the left side of the nucleus where the field remains high, providing the basis for further expansion of the conducting region into the depletion region in the form of a propagating impact ionization front.

The screening of the electric field, which is due to the collective dynamics of electron and holes, is the central feature of the breakdown mode under consideration here. It makes this mode essentially different from the conventional avalanche scenario of impact ionization breakdown, ${ }^{18}$ where electrons and holes drift apart during the ionization process.

At the moment when the impact ionization front is triggered, the extraction front has not necessarily reached the $n^{+}$ contact, and there can be a high concentration of electrons $n \approx N_{d}$ at the left side of the $n$ base. Therefore, in general, the impact ionization front and the extraction front can coexist in the system for some time.

\section{Propagation of the impact ionization front [Fig. 3(c), $1.60 \mathrm{~ns}<t<1.66 \mathrm{~ns}$ ]}

The third stage corresponds to the propagation of the impact ionization front whose velocity $v_{f}$ exceeds the saturated drift velocity $v_{s}$. For the parameters corresponding to Figs. 2 and 3 we obtain $v_{f} \approx 5 v_{s}$. The superfast front propagation is therefore a collective process not directly based on the drift motion of the individual carriers. The velocity $v_{f}$ $>v_{s}$ becomes possible due to the local multiplication of a small amount of mobile carriers present in the depleted region. These carriers are holes generated in the neutral region on the left that drift to the high field region on the right. The inner structure of the superfast front and the mechanism of its propagation are discussed in more detail in Sec. VI.

The current density and the maximum electrical field increase as the front propagates. Consequently, the plasma concentration behind the front also increases. Since the front velocity is much higher than the saturated drift velocity, the concentration profiles in the plasma behind the front remain frozen. Due to the interaction with the external load the voltage at the device decreases with increase of the current.
As the front propagates, the electrical field at the right side of the system starts to increase again due to the separation of the generated electrons and holes near the $p^{+}-n$ junction. Electrons move to the left and holes to right. This leads to the formation of a positively charged layer adjacent to the right boundary.

\section{Collision of the impact ionization front and the extraction front [Fig. 3(d), $1.66 \mathrm{~ns}<t<1.73 \mathrm{~ns}$ ]}

If all major carriers are removed from the system before the ionization front reaches the $n^{+}$contact, it continues to propagate up to the moment when the whole $n$ base is filled with a dense electron-hole plasma. Otherwise the ionization front and the extraction front collide. After the collision the character of the process changes due to the presence of a high concentration of major carriers in the area with strong electrical field: the ionization front passage is essentially terminated and we observe a quasi-uniform impact ionization in the neutral area. As the concentration increases, the electrical field on the left side of the structure decreases [Fig. 3(d), curves 1, 2, and 3]. For the chosen set of parameters this stage is not very well pronounced, since the extraction front is close to the $n^{+}$layer at the moment of collision. However, the situation becomes different in longer structures as well as for an earlier start of the impact ionization front caused by a higher voltage ramp applied to the device. Regimes with and without collision are both possible depending on the $n$-base length $W$, the voltage ramp $A$, and the initial bias $V_{0}$.

We should stress that generally the propagation of an ionization front generates a much higher concentration of free carriers than quasi-uniform breakdown. Indeed, during the fast propagation of an impact ionization front, a region of high electric field passes through the device and hence the ionization always develops in high electrical field. This results in high concentration of plasma behind the front. [A simple evaluation in the spirit of Ref. 22 gives $N_{d}\left(v_{f} / v_{s}\right)$ as a lower bound for plasma concentration.] In contrast, for uniform breakdown an increase of concentration is immediately followed by a decrease of electrical field due to the coupling to the external load, and high concentrations never can be reached. Therefore the collision between the two fronts should be avoided in applications.

After the switching the voltage at the device exhibits small amplitude oscillations (see Fig. 2). These oscillations are due to a sequence of impact ionization breakdowns near the right boundary of the $n$ base, in the vicinity of the $p^{+}-n$ junction. The mechanism of these breakdowns is as follows. As we mentioned in Sec. III C the voltage at the reversely biased $p^{+}-n$ junction starts to recover during the stage of front propagation. This happens due to the separation of electrons and holes near the $p^{+}-n$ junction. Whereas electrons leave this area drifting to the left, holes that drift to the right are constantly supplied by the plasma region. This leads to the formation of a positively charged region at the right side of the $n$ base. Due to the high concentration of holes $p$ $\gg N_{d}$, the slope of the electrical field $d E / d z=q(p$ $\left.+N_{d}\right) / \epsilon \epsilon_{0}$ in this region is orders of magnitude higher than in the depleted region. Thus the threshold of impact ioniza- 
tion $E_{a}$ at the right boundary can be reached again. As soon as it happens, an electron package, or an avalanche, is generated at the right boundary and the field again drops below the threshold. Due to the generated current pulse the voltage at the device decreases. The electrical field remains below the threshold during the drift of the electron package to the left. Then the field increases again and the next avalanche is generated. (This mechanism resembles the mechanism of IMPATT oscillations known in microwave electronics. ${ }^{18,19}$ ) This process is not sufficient for supporting the conduction state of the device and small amplitude oscillations are accompanied by a gradual increase of the average voltage.

\section{APPLICABILITY OF THE CONTINUUM APPROXIMATION}

We have shown that in unbiased structures the front is triggered by holes which are generated in the neutral layer and then drift through the depleted layer to the $p^{+}$contact, where the electrical field takes its maximum value. When modeling the triggering process in the continuum approximation, we face a crucial problem: the hole concentrations can be so small that the continuum approximation ceases to be valid. This problem manifests itself in the following artifact: impact ionization by electrons in the neutral layer generates holes with the unphysically small concentration $p \ll V^{-1}$, where $V$ is the system volume. This fraction of holes drift towards the $p^{+}$contact where their concentration grows exponentially in the high electrical field, reaches the physically relevant level $p \gg V^{-1}$, and eventually triggers the propagation of the impact ionization front. Consequently, the simulations predict that the front is triggered by any concentration of free carriers generated in the neutral area, even if this concentration corresponds to a small fraction of a hole in the complete volume of the device. This result might be either quantitatively or qualitatively wrong: in the first case the front is predicted to start too early and therefore it propagates at a lower voltage. In the second case, impact ionization in the neutral area is in fact totally incapable of triggering the front because the electrical field is insufficient. Thus straightforward modeling in the framework of the continuum approximation might lead to qualitatively wrong results. Since the velocity of the superfast front depends only logarithmically on the background ionization level in the depleted region, ${ }^{22}$ such simulations give plausible results for the front velocity and the switching time, and the mistake is hard to detect.

To overcome this problem, we propose the modified generation term [Eq. (10)] which incorporates the cutoff of the ionization reaction for unphysically low concentrations. The threshold concentrations $n_{\text {cut }}$ and $p_{\text {cut }}$ approximate the limit of validity of the continuum approximation. To check the relevance of such an approach, we have studied how the solutions depend on $n_{\text {cut }}$ and $p_{\text {cut }}$. The example described in the previous section has been calculated for $n_{\text {cut }}=p_{\text {cut }}$ $=10^{9} \mathrm{~cm}^{-3}$, which corresponds to an average distance between carriers $\sim 10 \mu \mathrm{m}$. In Fig. 4 this transient is shown again in curve 3 , while curves 1,2 , and 4 show the same process, but with $n_{\text {cut }}=p_{\text {cut }}=0,10^{6}$, and $10^{10} \mathrm{~cm}^{-3}$, respectively. Regardless of the cutoff level, the superfast switching

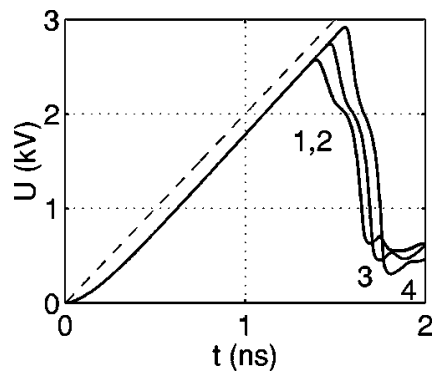

FIG. 4. Voltage at the device $U(t)$ calculated for different cutoffs $n_{\text {cut }}$, $p_{\text {cut }}=0,10^{6}, 10^{9}$, and $10^{10} \mathrm{~cm}^{3}$ (curves $1,2,3$, and 4 respectively). Curves 1 and 2 coincide within the accuracy of this graph. Other parameters as in Fig. 2.

remains qualitatively the same and the main phases of the process are the same. Quantitatively, however, for lower cutoff the switching process starts earlier, develops more slowly, and results in a higher residual voltage. All these effects are due to the earlier start of the impact ionization front that, in turn, leads to a slower propagation in a lower electrical field and a well-developed collision of ionization and extraction fronts. We conclude that although the continuum model is not able to provide accurate quantitative predictions, our results are essentially robust with respect to the cutoff level. In the case under consideration, the impact ionization in the neutral area is indeed sufficient for triggering the front.

In Fig. 5 we show the amount of holes generated in the neutral region as a function of the voltage ramp $A$ for different regimes. The hole concentration vanishes with decreasing $A$, especially in initially biased structures [Figs. 5(b) and 5 (c)], and the corresponding triggering mechanism ceases to exist. One of the important implications of Fig. 5 is that the minimal physical model evaluated in this article is not able to describe the switching of impact ionization fronts for the conditions of the original experiments: ${ }^{23-25}$ long ( $W$ $>200 \mu \mathrm{m})$ and initially biased structures $\left(V_{0}>400 \mathrm{~V}\right)$, and moderate voltage ramps $A \sim 1-2 \mathrm{kV} / \mathrm{ns}$. Section VI contains a further discussion of this problem.

\section{DEPENDENCE ON THE STRUCTURE AND CIRCUIT PARAMETERS}

\section{A. Influence of the system length}

In Fig. 6(a), the transient $U(t)$ is shown for different system lengths $W=50,100,150,200$, and $250 \mu \mathrm{m}$ (curves 1 , $2,3,4$, and 5, respectively). All other parameters are kept as in the basic example in Sec. III. With decrease of the system size the switching occurs earlier and develops faster. In shorter structures, the initial nucleus of electron-hole plasma becomes large compared to the system size $W$ and a substantial voltage drop takes place at the nucleation phase, whereas the stage of front propagation becomes less pronounced. As a result the fine structure of the $U(t)$ transient disappears, and the voltage at the device during the switching drops smoothly. Since the extraction front reaches the $n^{+}$contact earlier, the ionization front and the extraction front do not collide. Rather the ionization front propagates over the whole length of the $n$ base. This fact, together with the higher av- 

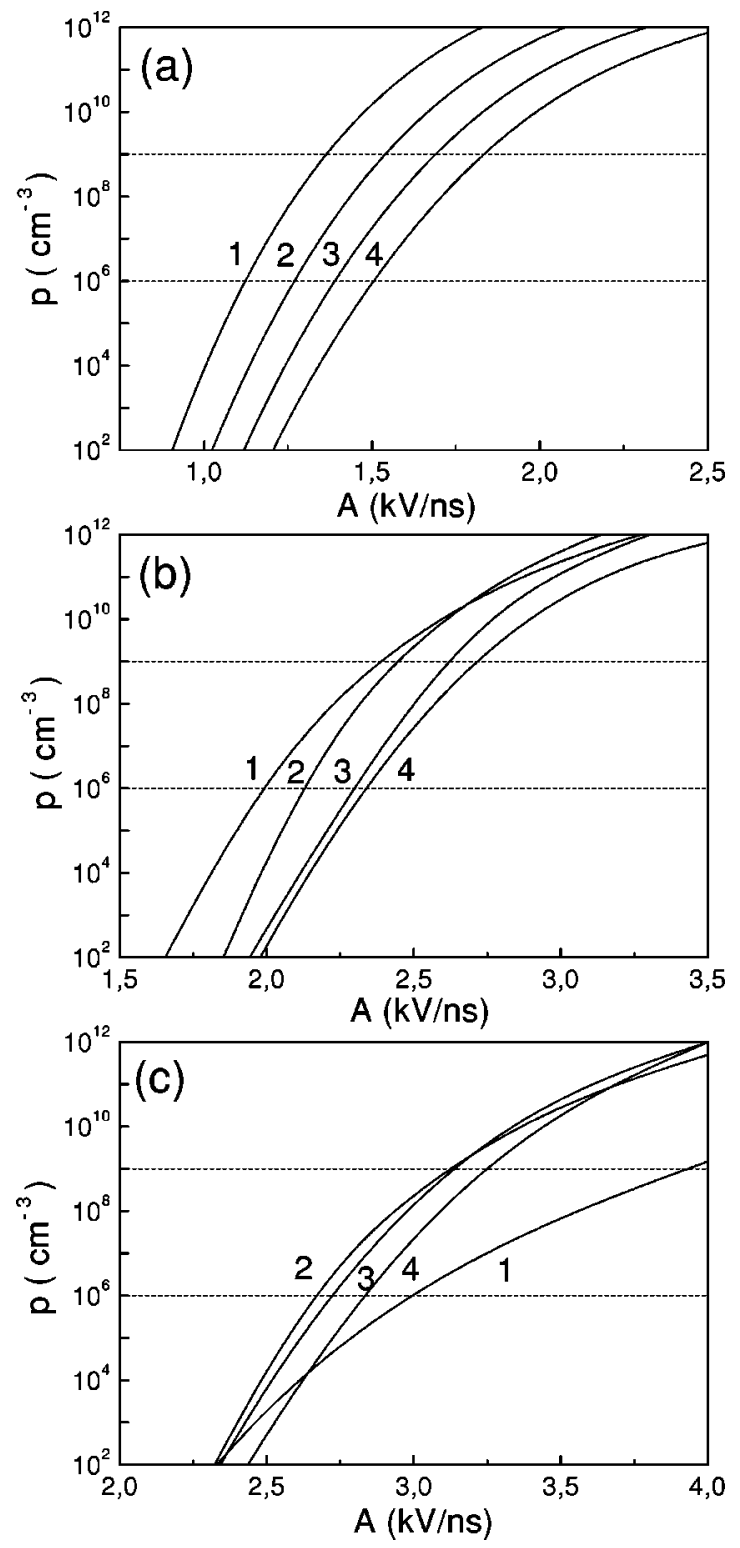

FIG. 5. The maximum concentration of holes generated by impact ionization in the neutral area as a function of the applied voltage $A$ for different initial biases $V_{0}$ and system lengths $W$. Panels (a), (b), and (c) correspond to the initial bias $V_{0}=0,400$, and $1000 \mathrm{~V}$, respectively. Curves $1,2,3$, and 4 correspond to the system lengths $W=150,200,250$, and $300 \mu \mathrm{m}$, respectively. Below the dashed line at $p=10^{6} \mathrm{~cm}^{-3}$ only few holes or fractions of holes are generated in the system (average distance between carriers $\sim 100$ $\mu \mathrm{m}$ ). Above the dashed line at $p=10^{9} \mathrm{~cm}^{-3}$ the concentration of holes is presumably sufficient for deterministic triggering of the impact ionization front (average distance between carriers $\sim 10 \mu \mathrm{m}$.

erage electrical field in a short structure, efficiently create a high conductivity throughout the whole structure and make the residual voltage as low as $70 \mathrm{~V}$ for $W=50 \mu \mathrm{m}$ [Fig. 6(a), curve 1].

In long structures the collision of ionization front and extraction front is unavoidable. Since the growth of conductivity by quasi-uniform ionization after this collision is less efficient than the previous process, the residual voltage increases and becomes as large as $800 \mathrm{~V}$ for $W=250 \mu \mathrm{m}$ [Fig. 6(a), curve 5]. Next, in presence of a thick neutral layer that acts as an internal ohmic resistance, the voltage on the device (a)

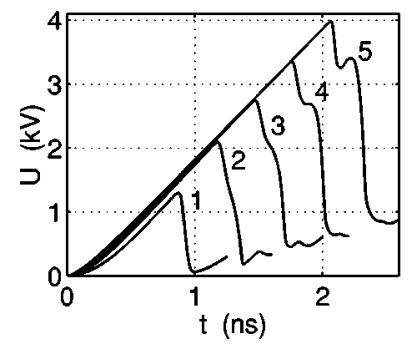

(b)

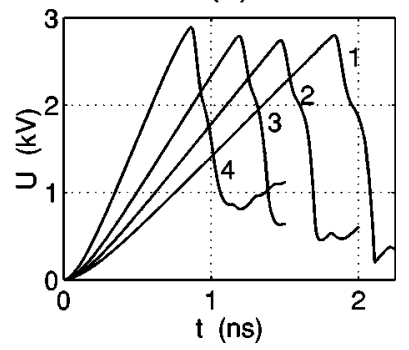

FIG. 6. (a) Voltage at the device $U(t)$ during the switching process calculated for different $n$-base lengths $W=50,100,150,200$, and $250 \mu \mathrm{m}$ (curves $1,2,3,4$, and 5 , respectively). (b) Voltage at the device $U(t)$ during the switching process for different voltage ramps $A=1.6,2.0,2.6$, and 4.0 $\mathrm{kV} / \mathrm{ns}$ (curves 1, 2, 3, and 4). Other parameters as in Fig. 2.

at the initial stage of front propagation even increases. As a result, the inflection of the $U(t)$ dependence (curve 3) that corresponds to the transition from the nucleation stage to the front propagation stage, grows further (curve 4), and the dependence can even become nonmonotonic (curve 5).

The maximum voltage at the device increases almost linearly from 1.3 to $4 \mathrm{kV}$ with system length. The voltage ramp during the switching $\left|(d U / d t)_{\text {down }}\right|$ has its maximum value of $10 \mathrm{kV} / \mathrm{ns}$ for $W=100,150 \mu \mathrm{m}$. It decreases with both an increase and decrease of the system length to values of 8.9 and $7.4 \mathrm{kV} / \mathrm{ns}$ for $W=50$ and $W=250 \mu \mathrm{m}$, respectively. In summary, shorter structures switch faster and have a lower residual voltage but commutate less power per pulse.

\section{B. Influence of the voltage ramp}

In Fig. 6(b) we present $U(t)$ transients for different voltage ramps while all other parameters are kept as in the basic example. The maximum voltage at the device at the moment of switching is about $\sim 2.8 \mathrm{kV}$ for all transients, whereas the delay time, the switching time, and the residual voltage are substantially different.

An increase of $A$ leads to a higher electrical field and a more efficient generation of initial carriers in the neutral layer. The ionization front is triggered earlier. Then, unavoidably, ionization front and extraction front collide. As a result, the residual voltage increases and reaches $\sim 800 \mathrm{~V}$ for $A$ $=4 \mathrm{kV} / \mathrm{ns}$ [Fig. 6(b), curve 4].

A decrease of the voltage ramp $A$ increases the delay of switching and ensures that the ionization front passes through the whole length of the $n$ base, so that the residual voltage is low ( $\sim 200 \mathrm{~V}$ for $A=1.6 \mathrm{kV} / \mathrm{ns})$. However, with a further decrease of $A$ we do not observe triggering any more since the electrical field in the neutral region region becomes too low to generate initial carriers.

\section{DISCUSSION}

\section{A. An alternative source of initial carriers}

We have shown above in Secs. III A, III B, and IV that if the voltage $\operatorname{ramp} A$ applied to an initially unbiased structure is sufficiently large, impact ionization in the neutral region is the dominant source of initial carriers. The high density $n$ $\approx N_{d}$ of electrons in the neutral region generates even in low 
fields a relatively high concentration of holes that will trigger the front after traveling through the $n$ base. Hence triggering can be considered as a deterministic process. Its time delay is determined by the generation time of holes in the neutral region and their travel time through the $n$ base. Our minimal model here appropriately captures the physical mechanism of the front triggering.

The behavior changes drastically when the applied voltage ramp $A$ is decreased and the initial bias $V_{0}$ is increased. The electrical field in the neutral region then becomes too low to generate a sufficient amount of holes and the front triggering fails. In Fig. 5 we show the maximum concentration of holes generated in the neutral region.

It is remarkable, that this triggering mechanism turns out to be ineffective for the parameters corresponding to the original experiments: ${ }^{23-25}$ high initial bias $V_{0}$ $\sim 600 \ldots 1000 \mathrm{~V}$, long $n$ base length $W \sim 220 \ldots 250 \mu \mathrm{m}$ and moderate voltage ramps $A \sim 1 \mathrm{kV} / \mathrm{ns}$. This result is in agreement with analytical estimates ${ }^{47}$ but in contradiction with earlier numerical simulations ${ }^{28,48,49}$ which have been performed in the framework of the same drift-diffusion model [Eqs. (1), (2), and (3)], but without cutoffs for low concentrations in Eq. (10). The analysis of the triggering stage shows that the switching observed in Refs. 48 and 49 is unphysical and is due to the multiplication of too small concentrations of mobile carriers, that either appear due to impact ionization in the neutral region or are assumed to be present in the depleted layer due to the thermal generation. These concentrations correspond to fractions of electrons and holes and can not be captured in a continuum approximation, as discussed in Sec. V. The numerical solutions from Refs. 48 and 49 can not be reproduced for any meaningful cut off $n_{\text {cut }}, p_{\text {cut }}$ in the generation term (10). Ref. 28 uses the same drift-diffusion model as Refs. 48 and 49, but it lacks information about the set of parameters used in simulations, and thus does not allow a comparison with our simulations.

This discrepancy with the experiments ${ }^{23-25}$ with initial bias indicates that another source of initial carriers becomes important. Thermal generation in the depleted layer can be excluded since the respective concentrations $n, p$ $\sim 10^{6} \mathrm{~cm}^{-3}$ (the estimate is based on the value $J$ $\sim 10^{-7} \mathrm{~A} / \mathrm{cm}^{2}$ of the thermally generated current in a reversely biased structure ${ }^{23}$ ) is too small and can not be captured within a continuum approximation. Thus the avalanche process would be stochastic and would therefore result in a local switching, but not in switching of the whole cross section of the device, ${ }^{50}$ in contrast to what is observed in experiments. ${ }^{23-25}$ An alternative source of initial carriers that could switch the device in a deterministic manner can be due to the standard manufacturing technology for such structures. Meanwhile this technology has been shown to have a side effect: ${ }^{51,52}$ it creates a specific type of process-induced deep level defects, coined as M-, U- and L-centers, ${ }^{51-54}$ with ionization energies $0.54,0.28$, and $0.34 \mathrm{eV}$, respectively, and concentrations up to $10^{14} \mathrm{~cm}^{-3}$. An important feature of these centers is a strong asymmetry between electron and hole capture cross sections. Due to the low cross section of hole capture, these centers do not assist the recombination and hence do not influence the lifetime of nonequilibrium
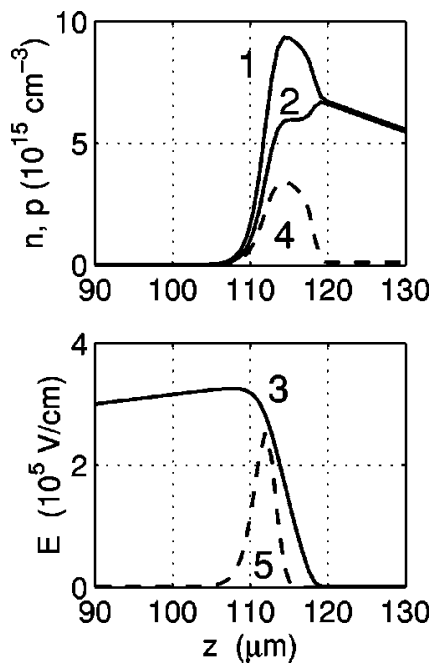

FIG. 7. The inner structure of the superfast impact ionization front. The concentrations of electrons and holes (curves 1 and 2 in the upper panel, respectively) and the electrical field (curve 3 in the lower panel) are shown at the some instant of time. The dashed lines show the difference of electron and hole concentration $(p-n)$ that is proportional to the space charge of mobile carriers (curve 4 in the upper panel) and the impact ionization rate $G$ (curve 5 in the lower panel, in arbitrary units).

carriers: one of the most important characteristics of the material in device application. Therefore their presence in the sample is generally not controlled in fabrication, and is even widely unknown. We propose that tunneling ionization with assistance of these deep centers represents the most probable mechanism of deterministic switching of initially biased structures for low voltage ramps. The corresponding model is presently under investigation.

\section{B. Plane superfast fronts and finger-like streamers}

The mechanism of superfast $\left(v_{f}>v_{s}\right)$ propagation of the plane ionization front in the layered structure is based on generation of electron-hole plasma by impact ionization followed by screening of the applied electrical field due to Maxwell relaxation. Due to the propagation of the front, the temporal sequence of plasma generation and screening becomes a spatial sequence. Fig. 7 shows the inner structure of the propagating front, the electric field profile, the concentration profiles, the generation term $G$ [Eq. (10)], and the space charge of the mobile carriers are shown at some instant of time. On the left, the electrical field increases due to the space charge of naked donors, while the space charge of mobile carriers is negligible. The impact ionization wave consists of an impact ionization region $(G>0$, see curve 5) followed by a region of electric screening (see curve 4). The position of the screening region roughly coincides with the concentration front: the edge of the plasma region. In the presence of pre-ionization (a small amount of mobile carriers in the depleted layer), the edge of the plasma region can move faster than the drift velocity of the individual carriers due to the local multiplication of existing carriers.

This propagation mechanism as well as the inner structure of the superfast front are quite general. A similar mechanism underlies the propagation of finger-shaped streamers in pre-ionized gases and solids. ${ }^{6,7,10-12}$ Recently superfast 
streamer fronts have also been found in numerical investigations of Corbino disks made from GaAs films. ${ }^{55,56}$ The essential ingredient for the formation of a superfast front in the presence of homogeneous pre-ionization is the spatial profile of the electrical field: the field that exceeds the threshold of impact ionization near the front should be below the threshold at a certain distance from the front. This provides a finite size of the "active zone," where the impact ionization develops, and prevents a uniform blowup of the concentration in the whole sample. ${ }^{15,16,57}$ For finger-like streamers, such a field profile is due to the Coulomb decrease of the electrical field with increasing distance from the space charge located at the streamer tip. ${ }^{11,12}$ For radially symmetrical fronts in Corbino disks, the decrease of the electrical field $E \sim r^{-1}$ results from the geometry of the concentric two-dimensional sample. ${ }^{3}$ For the plane fronts studied here, the electrical field decreases linearly $\left(d E / d z=q N_{d} / \epsilon \epsilon_{0}\right)$ due to the space charge of the naked donors in the depleted layer. Hence the superfast ionization front in the $p^{+}-n-n^{+}$structure should be regarded as a plane streamer-like front propagating into a charged low-conducting layer.

\section{Ionization fronts in bulk semiconductors}

Our numerical simulations show that superfast impact ionization fronts, which traditionally have been triggered in initially biased $p^{+}-n-n^{+}$structures, in fact can be triggered successfully by applying the voltage ramp to an equilibrium structure without initial bias. This finding indicates a new possibility to trigger similar superfast fronts in bulk samples without any $p-n$ junction. Indeed, originally layered $p^{+}-n-n^{+}$structures have been used mainly because the $p^{+}-n$ junction can support the initial voltage $V_{0}$. This ceases to be necessary when the device is operated without initial bias. Since all relevant processes develop inside the uniformly doped $n$ base, triggering and propagation of impact ionization front might be achieved in a bulk $n$-doped sample, as long as the contacts allow the extraction of electrons from the sample during the delay stage.

\section{SUMMARY}

A sharp voltage ramp applied to an initially unbiased layered $p^{+}-n-n^{+}$structure can initialize the propagation of a superfast ionization front that switches the structure to the high conductivity state within fractions of nanoseconds. It turns out that such a breakdown develops qualitatively in the same way as it has been believed to develop in heavily biased structures in the solitary-front-passage regime. The reason for this similarity is the delay in triggering due to the absence of initial carriers in the high field region. During this delay, the major carries can leave the $n$ base and form the depleted layer. Our findings indicate that similar planar ionization fronts can also be excited in plain bulk semiconductor samples without $p-n$ junctions.

In initially unbiased structures the front is triggered in a deterministic way by holes which, in turn, are generated by impact ionization in the neutral region of the structure during the delay stage. For increasing initial bias, this process eventually becomes inefficient. The minimal model, that takes only the band-to-band impact ionization into account, fails in this case. Impact ionization of deep impurity levels represents the most probable source of initial carriers in this regime.

The limits of the continuum approximation and the inherent presence of small densities of carriers, together of their important role in triggering the front, make the continuous drift-diffusion model insufficient for accurate quantitative description of the process. In certain cases this problem can be overcome by introducing cutoffs for small concentrations in the generation term.

Superfast plane fronts in semiconductor structures belong to the same family of streamer-like fronts as fingershaped streamers and plane streamer-like fronts in gases and solids in the presence of pre-ionization. The essential difference is that in layered doped structures, the front propagates not into a neutral but into a spatially charged depleted layer.

\section{ACKNOWLEDGMENTS}

Extended guest visits of one of the authors (P. R.) to Amsterdam have been supported by the Max-PlanckGesellschaft, by the Centrum voor Wiskunde en Informatica (CWI) in Amsterdam, and by the Dutch physics funding agency FOM. He was also supported by the Alexander von Humboldt Foundation. One of the authors (U. E.) was partially supported by the Netherlands Organization for Scientific Research NWO.

${ }^{1}$ Dynamics of Curved Fronts, edited by P. Pelcé (Academic, Boston, 1988).

${ }^{2}$ M. S. Cross and P. C. Hohenberg, Rev. Mod. Phys. 65, 851 (1993).

${ }^{3}$ E. Schöll, Nonlinear Spatio-temporal Dynamics and Chaos in Semiconductors (Cambridge University Press, Cambridge, 2000).

${ }^{4}$ H. Raether, Z. Phys. 112, 464 (1939) (in German); H. Raether, Electron avalanches and Breakdown in Gases (Butterworths, London, 1964).

${ }^{5}$ Electrical Breakdown in Gases, edited by J. M. Meek and J. D. Craggs (Wiley, New York, 1978).

${ }^{6}$ L. B. Loeb, Science 148, 1417 (1965).

${ }^{7}$ N. G. Basov, A. G. Molchanov, A. S. Nasibov, A. Z. Obidin, A. N. Pechenov, and Yu. M. Popov, Sov. Phys. JETP 43, 912 (1976) [Zh. Eksp. Teor. Fiz. 70, 1751 (1976)].

${ }^{8}$ E. M. Bazelyan and Yu. P. Raizer, Spark Discharges (CRS, New York, 1998).

${ }^{9}$ Yu. P. Raizer, Gas Discharge Physics (Springer, Berlin, 1997).

${ }^{10}$ S. K. Dhali and P. F. Williams, J. Appl. Phys. 62, 4696 (1987).

${ }^{11}$ M. I. D'yakonov and V. Yu. Kachorovskii, Sov. Phys. JETP 67, 1049 (1988). [Zh. Eksp. Teor. Fiz. 94, 321 (1988)].

${ }^{12}$ M. I. D'yakonov and V. Yu. Kachorovskii, Sov. Phys. JETP 68, 1070 (1989). [Zh. Eksp. Teor. Fiz. 95, 1850 (1989)].

${ }^{13}$ P. A. Vitello, B. M. Penetrante, and J. N. Bardsley, Phys. Rev. E 49, 5574 (1994).

${ }^{14}$ U. Ebert, W. van Saarloos, and C. Caroli, Phys. Rev. Lett. 77, 4178 (1996).

${ }^{15}$ U. Ebert, W. van Saarloos, and C. Caroli, Phys. Rev. E 55, 1530 (1997).

${ }^{16}$ U. Ébert and M. Arrayás, in Coherent Structures in Complex Systems (edited by D. Reguera et al.), Lecture Notes in Physics, Vol. 567 (Springer, Berlin, 2001), pp. 270-282.

${ }^{17}$ Electrical Discharges for Environmental Purposes: Fundamentals and Applications, edited by E. M. van Veldhuizen (NOVA Science, New York, 1999).

${ }^{18}$ S. M. Sze, Physics of Semiconductor Devices (Wiley, New York, 1981).

${ }^{19}$ M. Shur, Physics of Semiconductor Devices (Prentice-Hall, Englewood Cliffs, 1990).

${ }^{20}$ M. Shaw, V. Mitin, E. Schöll, and H. Grubin, The Physics of Instabilities in Solid State Electron Devices (Plenum, New York, 1992).

${ }^{21}$ H. J. Prager, K. K. N. Chang, and J. Wiesbord, Proc. IEEE 55, 586 (1968). 
${ }^{22}$ B. C. Deloach and D. L. Scharfetter, IEEE Trans. Electron Devices ED20, 9 (1970)

${ }^{23}$ I. V. Grekhov, A. F. Kardo-Sysoev, L. S. Kostina, and S. V. Shenderei, Electron. Lett. 17, 422 (1981); Tech. Phys. 26, 984 (1981) [Pis'ma Zh. Tekh. Fiz. 51, 1709 (1981)].

${ }^{24}$ D. Benzel and M. Pocha, Rev. Sci. Instrum. 56, 1456 (1985).

${ }^{25}$ I. V. Grekhov and A. F. Kardo-Sysoev, Sov. Tech. Phys. Lett. 5, 395 (1979) [Pis'ma Zh. Tekh. Fiz. 5, 950 (1979)].

${ }^{26}$ I. V. Grekhov, Solid-State Electron. 32, 923 (1989).

${ }^{27}$ A. F. Kardo-Susoev, V. M. Efanov, and I. G. Chashnikov, in Digest of Technical Papers, 10th IEEE International Pulsed Power Conference, edited by W. L. Baker and G. Cooperstain, Albuquerque, N.M 1995, pp. 342-347.

${ }^{28}$ R. J. Focia, E. Schamiloglu, C. B. Fledermann, F. J. Agee, and J. Gaudet, IEEE Trans. Plasma Sci. 25, 138 (1997).

${ }^{29}$ Let us mention also two other important differences: (i) in a TRAPATT diode the impact ionization front starts when the $n$ base is fully depleted (no major carriers), whereas in large-scale structures the $n$ base is only partially depleted. This changes the longitudinal dynamics of the ionization front due to its interaction with the extraction front (see Sec. III). (ii) due the large cross sections, transverse instabilities of plane propagating fronts and current localization may develop.

${ }^{30}$ S. N. Vainstein, Zu. V. Zhilyaev, and M. E. Levinstein, Sov. Tech. Phys. Lett. 14, 664 (1988).

${ }^{31}$ A. Minarsky and P. Rodin, Sov. Tech. Phys. Lett. 20, 490 (1994) [Pis'ma Zh. Tekh. Fiz. 20, 38 (1994)].

${ }^{32}$ A. Minarsky and P. Rodin, Solid-State Electron. 41, 813 (1997).

${ }^{33}$ A. Minarsky and P. Rodin, Semiconductors 31, 366 (1997) [Fiz. Tekh. Poluprovodn. (S.-Peterburg) 31, 432 (1997)].

${ }^{34}$ H. Jalali, R. Joshi, and J. A. Gaudet, IEEE Trans. Electron Devices 45, 1761 (1998).

${ }^{35}$ I. V. Grekhov, V. M. Efanov, A. F. Kardo-Susoev, and I. A. Smirnova, Sov. Tech. Phys. Lett. 11, 372 (1985) [Pis'ma Zh. Tekh. Fiz. 11, 901 (1985)].

${ }^{36}$ V. A. Kozlov, A. F. Kardo-Susoev, and V. I. Brulevsky, Semiconductors 35, 608 (2001) [Fiz. Tekh. Poluprovodn. (S.-Peterburg) 35, 629 (2001)].

${ }^{37}$ Zh. I. Alferov, I. V. Grekhov, V. M. Efanov, A. F. Kardo-Susoev, V. I. Korol'kov, and M. N. Stepanova, Sov. Tech. Phys. Lett. 13, 454 (1987) [Pis'ma Zh. Tekh. Fiz. 13, 1089 (1987)].

${ }^{38}$ I. V. Grekhov and V. M. Efanov, Sov. Tech. Phys. Lett. 14, 920 (1988) [Pis'ma Zh. Tekh. Fiz. 14, 2121 (1988)].

${ }^{39}$ I. V. Grekhov and V. M. Efanov, Sov. Tech. Phys. Lett. 16, 645 (1990) [Pis'ma Zh. Tekh. Fiz. 16, 9 (1990)].

${ }^{40}$ The results of our simulations are not sensitive to boundary conditions. We observe the same picture of front triggering and propagation even for
Neumann boundary conditions for concentrations, though the residual voltage becomes somewhat lower.

${ }^{41}$ A. Wacker and E. Schöll, J. Appl. Phys. 78, 7352 (1995).

${ }^{42}$ C. Jacobini, C. Canali, G. Ottaviani, and A. Alberigi, Solid-State Electron. 20, 77 (1977).

${ }^{43}$ We note that the results of our simulations are essentially independent of the diffusion coefficient. Since this is not obvious a priori for all stages of the process, we have incorporated the diffusion term in our model.

${ }^{44}$ V. A. Kuz'min, N. N. Krukova, A. S. Kuregyan, T. T. Mnatsakanov, and V. B. Shuman, Sov. Phys. Semicond. 9, 481 (1975) [Fiz. Tekh. Poluprovodn. (S.-Peterburg) 9, 735 (1975)].

${ }^{45}$ J. C. Strikwerda, Finite Difference Schemes and Partial Differential Equations (Chapman \& Hall, New York, 1989).

${ }^{46} \mathrm{~J}$. G. Verwer, W. Hundsdorfer, and J. G. Blom, Numerical Time Integration for Air Pollution Models, Surveys on Mathematics for Industry, Vol. 10 (2002) (Preprint: CWI Report MAS-R9825, CWI, Amsterdam.)

${ }^{47}$ I. V. Grekhov, A. F. Kardo-Sysoev, M. V. Popova and S. V. Shenderei, Sov. Phys. Semicond. 17, 877 (1983) [Fiz. Tekh. Poluprovodn. (S.-Peterburg) 17, 1380 (1983)].

${ }^{48}$ Yu. D. Bilenko, M. E. Levinstein, M. V. Popova, and V. S. Yuferev, Sov. Phys. Semicond. 17, 1156 (1983) [Fiz. Tekh. Poluprovodn. (S.-Peterburg) 17, 1812 (1983)].

${ }^{49}$ A. F. Kardo-Sysoev and M. V. Popova, Semiconductors 30, 431 (1996) [Fiz. Tekh. Poluprovodn. (S.-Peterburg) 30, 803 (1996)]

${ }^{50}$ The inverse frequency of thermal generation in the depleted layer-of the order of $1 \mathrm{~ns}$ at room temperature-imposes a limitation on the duration of the latent stage (delay in triggering) that can not exceed several nanoseconds. If the front is not triggered deterministically within this time but the applied voltage continues to increase, thermal carriers will initialize an irregular avalanche breakdown, it is likely to lead to current localization and thermal destruction.

${ }^{51}$ E. V. Astrova, V. B. Voronkov, V. A. Kozlov, and A. A. Lebedev, Semicond. Sci. Technol. 13, 488 (1998).

${ }^{52}$ E. V. Astrova, V. A. Kozlov, A. A. Lebedev, and V. B. Voronkov, Solid State Phenom. 69-70, 539 (1999).

${ }^{53}$ L. D. Yau and C. T. Sah, Solid-State Electron. 17, 193 (1974).

${ }^{54}$ C. T. Sah and C. T. Yang, J. Appl. Phys. 6, 1767 (1975).

${ }^{55}$ G. Schwarz, C. Lehmann, and E. Schöll, Phys. Rev. B 61, 10 194(2000).

${ }^{56}$ G. Schwarz, Ph.D. Thesis, Institute of Theoretical Physics, TU Berlin, 2000 (URL: http://edocs.tu-berlin.de/diss/2001/schwarz_georg.pdf)

${ }^{57}$ Note, that the spatial structure of a propagating front also can be created by a spatially decaying pre-ionization in a constant electric field in a planar geometry. 